\title{
LESÕES PROVENIENTES DE PROCEDIMENTO CIRÚRGICO: FATORES RELACIONADOS
}

\author{
Lesions arising out of surgical procedure: related factors
}

\section{Lesiones provenientes de procedimiento quirúrgico: factores relacionados}

\author{
Sílvia Márcia dos Santos Sandes ${ }^{* *}$ (), Mikael Ferreira $\operatorname{Costa}^{2}$ (D), Graycielle Vieira dos $\operatorname{Santos}^{3}$ (), Lucas Pereira de Freitas ${ }^{4}$ (D), \\ Alessa Caroline Pedroza de Vasconcelos ${ }^{5}$ (1) , Luciana de Santana Lôbo Silva ${ }^{6}$ (1)
}

RESUMO: Objetivos: Analisar as publicações científicas referentes a lesões de pele decorrentes de procedimento cirúrgico e identificar os fatores de risco associados à ocorrência das lesões. Método: Estudo de revisão integrativa de literatura, com publicações do período de 2012 a 2018 , utilizando as bases das bibliotecas PubMed e Biblioteca Virtual de Saúde. Resultados: Dos oito estudos que contemplaram o objetivo, 87,5\% foram pesquisas desenvolvidas em instituições hospitalares e 12,5\% em centro médico; e 25,0\% dos estudos utilizaram o método de caso controle, 25,0\% eram estudos de análise retrospectiva, 12,5\% eram estudo de seleção de amostra, 12,5\% estudo transversal, 12,5\% relato de caso e 12,5\% estudo de coorte. Das publicações, 50,0\% foram em revistas médicas, 12,5\% em revistas de enfermagem e 37,5\% em revistas de outras áreas da saúde. Considerações finais: As lesões que acontecem apor conta de procedimento cirúrgico, em sua maioria, são relacionadas à pele, tanto no intraoperatório quanto no pós-operatório imediato. Além das lesões por pressão, destacaram-se a presença de queimaduras no intraoperatório e o aparecimento de lesões de pele no período pós-operatório. Palavras-chave: Lesão por pressão. Ferimentos e lesões. Queimaduras. Salas cirúrgicas. Posicionamento do paciente.

ABSTRACT: Objectives: To analyze scientific publications regarding skin lesions resulting from surgical procedures and to identify the risk factors associated with their occurrence. Method: Integrative literature review study, with publications from 2012 to 2018, using PubMed Library and Virtual Health Library databases. Results: Of the eight studies that contemplated the subject, $87.5 \%$ were researches developed in hospital institutions and $12.5 \%$ in medical centers; $25.0 \%$ of the studies used case-control method, $25.0 \%$ were retrospective analysis studies, $12.5 \%$ were sample selection studies, $12.5 \%$ were cross-sectional studies, $12.5 \%$ were case reports and $12.5 \%$ were cohort studies. In total, $50.0 \%$ had been published in medical journals, $12.5 \%$ in nursing journals and $37.5 \%$ in journals of other health areas. Final considerations: The lesions that occur due to surgical procedures are mostly related to skin, both intraoperatively and in the immediate postoperative period. In addition to pressure ulcers, the presence of intraoperative burns and the appearance of skin lesions in the postoperative period were noted.

Keywords: Pressure ulcer. Wounds and injuries. Burns. Operating rooms. Patient positioning.

RESUMEN: Objetivos: Analizar las publicaciones científicas referentes a lesiones de piel decurrentes de procedimiento quirúrgico e identificar los factores de riesgo asociados a la ocurrencia de las lesiones. Método: Estudio de revisión integrativa de literatura, con publicaciones del período de 2012 a 2018 , utilizando las bases de las bibliotecas PubMed y Biblioteca Virtual de Salud. Resultados: De los ocho estudios que contemplaron el objetivo, un 87,5\% fue investigación desarrollada en instituciones hospitalarias y un 12,5\% en centro médico; y un 25,0\% de los estudios utilizaron el método de caso control, un $25,0 \%$ era estudio de análisis retrospectivo, un 12,5\% era estudio de selección de muestra, un 12,5\% estudio transversal, un $12,5 \%$ relato de caso y un $12,5 \%$ estudio de cohorte. De las publicaciones, un $50,0 \%$ fue en revistas médicas, un $12,5 \%$ en revistas de enfermería y un $37,5 \%$ en revistas de

\footnotetext{
'Enfermeira; mestre em Ciências Fisiológicas pela Universidade Federal de Sergipe (UFS). Docente do curso de Enfermagem da Faculdade Estácio de Sergipe - Aracaju (SE), Brasil. ²Enfermeiro; mestrando pelo Programa de Pós-Graduação em Biologia Parasitária da UFS - Aracaju (SE), Brasil.

${ }^{3}$ Acadêmica de Enfermagem pela Faculdade Estácio de Sergipe - Aracaju (SE), Brasil.

${ }^{4}$ Acadêmico de Enfermagem pela Faculdade Estácio de Sergipe - Aracaju (SE), Brasil.

${ }^{5}$ Farmacêutica; Doutora em Biotecnologia pela Rede Nordeste de Biotecnologia; mestre em Saúde e Ambiente pela Universidade Tiradentes; d. Docente do curso de Enfermagem da Faculdade Estácio de Sergipe - Aracaju (SE), Brasil.

${ }^{6}$ Enfermeira; mestre em Enfermagem pela UFS; docente do curso de enfermagem da Faculdade Estácio de Sergipe - Aracaju (SE), Brasil.

*Autor correspondente: sil.sandes@hotmail.com

Recebido: 23/08/2018 - Aprovado: 01/06/2019

DOI: $10.5327 / 21414-4425201900030008$
} 
otras áreas de la salud. Consideraciones finales: Las lesiones que ocurren por cuenta de procedimiento quirúrgico, en su mayoría, son relacionadas a la piel, tanto en el intraoperatorio como en el posoperatorio inmediato. Además de las lesiones por presión, se destacaron la presencia de quemaduras en el intraoperatorio y la aparición de lesiones de piel en el período posoperatorio.

Palabras clave: Úlcera por presión. Heridas y traumatismos. Quemaduras. Quirófanos. Posicionamiento del paciente.

\section{INTRODUÇÃo}

Na última década, a segurança do paciente tornou-se destaque nas discussões de cuidados com pessoas e nos processos que as envolvem. Diversos estudos têm buscado melhores práticas para obter segurança, bem como instituições que visam constantemente à melhoria em seus processos com as certificações de acreditação. O Centro Cirúrgico (CC), por sua complexidade, merece um olhar diferenciado no cuidado do paciente, com atuação de diferentes profissionais e integração de várias unidades; sua especificidade exige atenção nos processos que cercam o paciente ${ }^{1}$.

O CC de um hospital é o lugar onde ocorre a maior parte dos eventos adversos à saúde dos pacientes. Nele, são executados procedimentos terapêuticos e diagnósticos de pequena, média e alta complexidade, que requerem um processo de trabalho complexo e interdisciplinar, pois as intervenções cirúrgicas integram a assistência à saúde, contribuindo para a prevenção de agravos à integridade física e à perda de vidas. Muitas vezes, os CCs são associados aos riscos de complicações e morte ${ }^{2}$.

Nesse contexto, o posicionamento cirúrgico é um passo essencial e, com frequência, subestimado. Todavia, há que se considerar que pode levar a lesões graves, mas evitáveis ${ }^{3}$. O paciente fica totalmente dependente da equipe para implementação de cuidados, de modo a impedir ou minimizar problemas relacionados ao posicionamento. Já a equipe deve proteger a integridade neuromuscular e cutânea, manter o alinhamento corporal e as funções respiratória e circulatória, principalmente ${ }^{4}$.

Todos os membros da equipe cirúrgica (enfermeiro, circulante de sala, anestesiologista, cirurgião e assistentes) são responsáveis pelo posicionamento do paciente à cirurgia. Portanto, devem estar envolvidos na identificação de riscos, na manutenção da segurança do paciente e, por conseguinte, na proteção de possíveis eventos adversos durante a cirurgia 5 .

A permanência prolongada na mesma posição pode ocasionar risco potencial para alterações anatômicas e fisiológicas, danos às articulações, estiramentos, esforço muscular, lesões nervosas ou luxações ${ }^{6}$, podendo levar à dor musculoesquelética, lesões de pele, de nervos periféricos e à síndrome compartimental ${ }^{4}$.
Dessa forma, o interesse para o desenvolvimento desta pesquisa surgiu na disciplina de Ensino Clínico em Cirurgia, por ocasião do trabalho sobre indicadores de qualidade, conduzindo os pesquisadores à seguinte questão norteadora: Quais são as lesões relacionadas ao procedimento cirúrgico descritas na literatura?

\section{OBJETIVOS}

- Analisar as publicações científicas referentes a lesões de pele decorrentes de procedimento cirúrgico;

- Identificar os seguintes fatores de risco associados à ocorrência das lesões: tipo de anestesia, procedimento realizado e duração da cirurgia.

\section{MÉTODO}

Trata-se de um estudo retrospectivo, do tipo revisão integrativa da literatura, com recorte temporal de seis anos. Para a elaboração da revisão, foram fundamentadas as seguintes etapas: definição da pergunta norteadora, objetivos, busca de evidências na literatura, estabelecimento de critérios de inclusão e exclusão de artigos, análise, checagem, discussão e apresentação dos resultados ${ }^{7}$.

A questão norteadora da pesquisa foi elaborada pela estratégia PICO (acrônimo para Patient, Intervention, Comparison, Outcomes $)^{8}$. Assim, a pergunta de pesquisa delimitada foi: Quais são as lesões relacionadas ao procedimento cirúrgico descritas na literatura? As bases utilizadas foram a U.S. National Library of Medicine (PubMed) e a Biblioteca Virtual em Saúde (BVS).

Os critérios de inclusão foram: artigos originais publicados em português e inglês disponíveis eletronicamente em texto completo, com nível de evidência para ensaios clínicos randomizados controlados; e estudos observacionais e relatos de caso que abordassem lesões relacionadas ao procedimento cirúrgico, intervenções e / ou cuidados de prevenção, no período de 2012 a 2018. A pesquisa buscou obter artigos 
mais atualizados, uma vez que a ciência se encontra em constante renovação. Foram excluídos estudos de revisão e metanálises, dissertações, teses e editoriais.

Durante a pesquisa dos artigos, foi adaptado o modo de busca de acordo com a particularidade de cada base de dados, utilizando os seguintes descritores em saúde:

- Lesão por pressão;

- Ferimentos e lesões;

- Queimaduras;

- Salas cirúrgicas;

- Posicionamento do paciente (Quadro 1).

Com o intuito de selecionar os artigos para a revisão, foi elaborada uma estratégia de análise, obedecendo à questão norteadora e aos critérios de inclusão e exclusão. Tal análise se deu por meio da leitura de títulos, resumos e textos completos e resultou em seis artigos. Após essa etapa, foram excluídos os artigos replicados (Figura 1).

Para a análise e posterior discussão dos artigos, fez-se um quadro sinóptico, desenvolvido pelos pesquisadores, que contemplou os seguintes aspectos considerados relevantes: nome dos autores, lesões descritas, caracterização da amostra e conclusões. Os dados foram processados por meio dno Microsoft Office Excel 2010, seguidos da estatística descritiva e apresentados em forma de figura e quadro.

\section{RESULTADOS}

No presente estudo, foram encontrados 20 artigos sobre o tema proposto, no entanto analisaram-se oito, que contemplaram os critérios de inclusão estabelecidos. Destes, sete (87,5\%) foram da PubMed e um (12,5\%) da BVS.

Dos artigos selecionados, sete $(87,5 \%)$ consistiram em pesquisas desenvolvidas em instituições hospitalares e um $(12,5 \%)$ em um centro médico.
Com relaçãoNo que tange ao método de pesquisa, foram dois $(25,0 \%)$ estudos caso controle, dois $(25,0 \%)$ estudos de análise retrospectiva, um (12,5\%) estudo de seleção de amostra, um $(12,5 \%)$ estudo transversal, um (12,5\%) relato de caso e um (12,5\%) estudo de coorte.

A respeito do tipo de periódico no qual os artigos inseridos na pesquisa foram publicados, quatro $(50,0 \%)$ apareceram em revistas médicas, um $(12,5 \%)$ em revista de enfermagem e três $(37,5 \%)$ em revistas de outras áreas da saúde.

Em relação aos anos de publicação referentes ao número de publicações selecionadas no estudo, o corte temporal foiabrangeu de 2012 a 2018. Evidenciou-se maior percentual de publicações entre os anos 2013 e 2015, com dois artigos $(25,0 \%)$ em cada ano (Figura 2).

Os tipos de especialidades cirúrgicas envolvidas nas pesquisas analisadas subdividem-se em cirurgia geral, cardíaca, ortopédica, torácica, neurológica e plástica. São todos estudos de pacientes submetidos a procedimentos cirúrgicos, com amostras variando entre 143 e 32.963 sujeitos. O Quadro 2 apresenta um resumo dos resultados dessa revisão integrativa.

\section{DISCUSSÃO}

Em relação ao objetivo desta revisão, observaram-se, nos oito artigos selecionados, algumas variantes que estavam associadas diretamente às lesões provenientes do procedimento cirúrgico, como o procedimento realizado, a duração da cirurgia e o tipo de anestesia.

A pesquisa em busca das lesões acometidas ao paciente cirúrgico é de suma importância para chamar a atenção para o fato de que a equipe cirúrgica deve estabelecer cuidados para preservar a integridade física dos pacientes.

Em um estudo sobre as lesões perioperatórias foram destacadas as queimaduras e as lesões por pressão. Os autores relatam

Quadro 1. Distribuição das estratégias de busca, segundo base de dados e número de artigos localizados.

\begin{tabular}{|l|l|c|c|}
\hline Bases & \multicolumn{1}{c|}{ Busca } & $\begin{array}{c}\text { Artigos } \\
\text { resgatados }\end{array}$ & $\begin{array}{c}\text { Artigos } \\
\text { aproveitados }\end{array}$ \\
\hline PubMed & $\begin{array}{l}\text { Pressure Ulcer AND Patient positioning; Wounds and Injuries AND Patient } \\
\text { positioning; Burns and Patient Positioning; Pressure Ulcer AND Operating rooms; } \\
\text { Wounds and injuries AND Operating rooms; Burns AND Operating rooms }\end{array}$ & 14 \\
\hline BVS & $\begin{array}{l}\text { Lesão por pressão AND Salas cirúrgicas; Ferimentos e lesões AND Salas } \\
\text { cirúrgicas; Queimaduras AND Salas cirúrgicas; Lesão por pressão AND }\end{array}$ & 6 \\
\hline $\begin{array}{l}\text { Posicionamento do paciente; Ferimentos e lesões AND Posicionamento do } \\
\text { paciente; Queimaduras AND Posicionamento do paciente }\end{array}$ & 1 \\
\hline
\end{tabular}

BVS: Biblioteca Virtual em Saúde. 
que $10,7 \%$ dos pacientes, do total de 2.695 , adquiriram lesões por pressão (LP) após o procedimento cirúrgico ${ }^{12}$.

Outras investigações relatam as lesões de pele provenientes do procedimento cirúrgico relacionadas ao tipo de cirurgia enfrentada pelo paciente, no entanto os autores não apontam o tipo de cirurgia com maior incidência de lesões. Entre as cirurgias elencadas pelos autores, destacam-se as especialidades: cardiovascular, torácica, ortopédica, neurológica, plástica, urológica, bariátrica, geral, hepatobiliar, oncológica, trauma, transplante e vascular. As lesões vão desde integridade da pele e tissular prejudicada, considerada estágio I de lesão por pressão, até lesão por pressão de estágio IV $^{9,11-13}$.

Além das LP evidenciadas pelos autores, outros pesquisadores abordam que estas estão diretamente relacionadas a queimaduras ocorridas durante procedimentos cirúrgicos. O maior índice de queimaduras está ligado ao dispositivo de eletrocautério, que, na maioria dos casos, é o ponto de partida para queimaduras ${ }^{10,17}$.
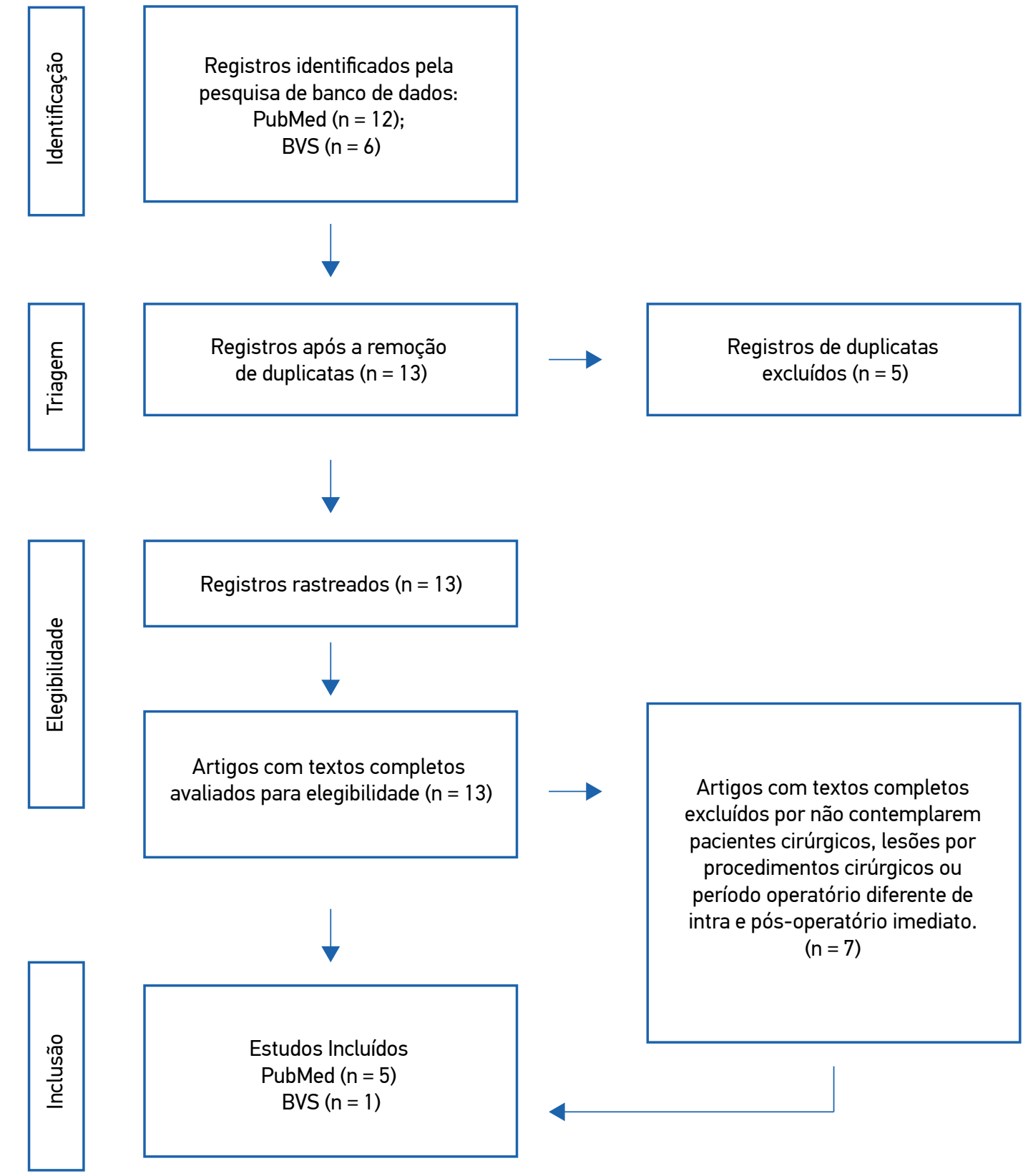

BVS: Biblioteca Virtual em Saúde.

Figura 1. Fluxograma para seleção dos artigos incluídos na revisão. 
Uma pesquisa identificou que $90 \%$ das queimaduras foram causadas pelo eletrocautério ${ }^{17}$.

Outra característica importante está vinculada à presença dos componentes essenciais para o início de incêndio, que são encontrados de forma abundante no CC. Os três itens básicos são o oxidante, a fonte de ignição e o combustível. O oxidante relatado pelos autores foi o oxigênio, já que por conta de procedimentos cirúrgicos complexos e dos altos níveis de sedação são ofertadas altas concentrações de oxigênio ${ }^{10,17}$. Os autores entendem que, em sua maioria, as queimaduras no CC poderiam ser evitadas, se tomadas as medidas de precaução adequadas e eficazes.

Quanto aos tempos operatórios, verificou-se que, logo após a cirurgia, já é possível identificar LP e isso se dá em função do tempo prolongado de imobilidade e da pressão exercida em determinadas áreas do corpo do paciente. Identificou-se alto índice de pacientes com lesões após procedimentos com períodos mais extensos. Dos 297 pacientes acompanhados, 44 apresentaram LP, com taxa de 9,8\% de lesões encontradas nos primeiros 30 minutos após a cirurgia, e $5,1 \%$ dos pacientes com lesões após os primeiros 30 minutos pós-cirurgia ${ }^{11,13}$.

Houve maior incidência de lesões em pacientes com tempo de cirurgia prolongado (período igual ou superior a 4 horas), com média de $4,5 \%$ lesões encontradas em pacientes nas primeiras 24 horas após a cirurgia ${ }^{11}$.

Quanto maior o porte da cirurgia, maior é o risco de o indivíduo adquirir lesão, já que, para cirurgias mais complexas, é determinado um tempo cirúrgico mais extenso. Também, sob anestesia geral, na qual o paciente fica imóvel por um grande período de tempo, são administrados anestésicos mais potentes ${ }^{11,13}$. É recomendada mais atenção no período pós-operatório, em razão de a incidência de LP ser notável nesse período ${ }^{12}$.

No que se refere ao tipo de anestesia, a geral é a mais associada a lesões de pele. Pacientes submetidos à anestesia geral tiveram variação 4,8 vezes maior, quando comparados com os pacientes nos quais foi realizada anestesia local ${ }^{13}$.

Os pacientes em uso de anestesia geral estão mais expostos a sofrerem queimaduras, pois a taxa de sedação é maior, em relação a outros fármacos anestésicos, o que, consequentemente, exige maior taxa de oxigênio, queéo componente em potencial para incêndios ${ }^{17}$.

Autores de um estudo relataram dificuldades em abordar esse dado, uma vez que a pesquisa era voltada a pacientes críticos, o que influenciava diretamente o uso de anestésicos gerais em cirurgias prolongadas, ligadas à situação clínica dos pacientes $^{12}$. Os demais autores não trazem associação entre o agente anestésico e a ocorrência de lesões.

\section{CONSIDERAÇÕES FINAIS}

Diante da realização deste estudo, foi possível conhecer, pela literatura analisada, a incidência de lesões consequentes de procedimentos cirúrgicos. As mais comuns são as lesões por

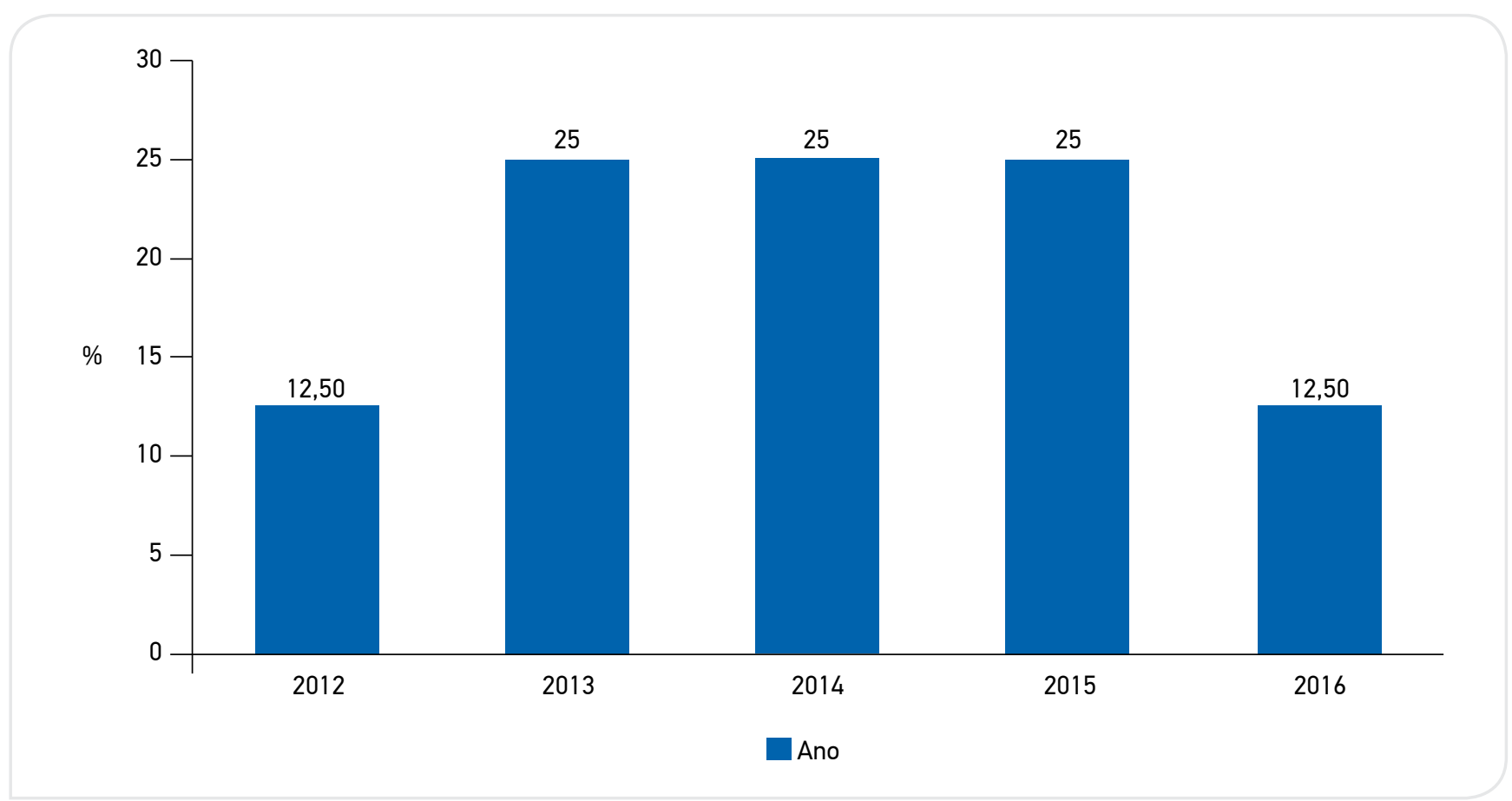

Figura 2. Quantitativo de publicações por ano, com corte temporal de 2012 a 2018. 
Quadro 2. Síntese das pesquisas incluídas na revisão integrativa.

\begin{tabular}{|c|c|c|c|}
\hline Autores, país e ano & Lesões descritas & Caracterização da amostra & Conclusões \\
\hline $\begin{array}{l}\text { Steyer et al. } \\
\text { (Brasil, 2016) }^{9}\end{array}$ & $\begin{array}{l}\text { Integridade tissular } \\
\text { da pele prejudicada }\end{array}$ & $\begin{array}{l}\text { Dos } 143 \text { pacientes submetidos à cirurgia } \\
\text { bariátrica, } 133 \text { tiveram a integridade tissular } \\
\text { da pele prejudicada. }\end{array}$ & $\begin{array}{l}\text { A escassez de estudos de } \\
\text { enfermagem sobre cirurgia bariátrica } \\
\text { trouxe limitação à discussão dos } \\
\text { dados, corroborando a necessidade de } \\
\text { ampliar investigações sobre o tema. }\end{array}$ \\
\hline $\begin{array}{l}\text { Jalali et al. } \\
(\text { Irã, 2015) }\end{array}$ & Lesão térmica & $\begin{array}{l}\text { Foram selecionados } 300 \text { pacientes submetidos } \\
\text { à cirurgia cardíaca, divididos em dois grupos: } \\
150 \text { antes das modificações da engenharia e } \\
150 \text { após as modificações. Foram identificados } \\
110 \text { pacientes com queimaduras: } 75 \text { antes e } 35 \\
\text { depois das modificações. }\end{array}$ & $\begin{array}{l}\text { A vigilância e os reparos de defeitos } \\
\text { por profissionais especializados } \\
\text { trouxeram efeitos significativos na } \\
\text { redução da incidência de queimaduras. }\end{array}$ \\
\hline $\begin{array}{l}\text { Hayes et al. (Estados } \\
\text { Unidos, 2015) }\end{array}$ & Lesão por pressão & $\begin{array}{l}\text { Estudo com } 32.963 \text { pacientes que não } \\
\text { possuíam lesões no ato da admissão, } \\
\text { submetidos às cirurgias cardíaca, geral, } \\
\text { hepatobiliar, neurológica, oncológica, plástica, } \\
\text { torácica, transplante, trauma, urológica e } \\
\text { vascular. Foram documentadas } 931 \text { LP. }\end{array}$ & $\begin{array}{l}0 \text { tempo cirúrgico é um fator de } \\
\text { risco para o desenvolvimento de LP. } \\
\text { A maioria das LP não aparece no pós- } \\
\text { operatório imediato, e os esforços de } \\
\text { prevenção devem concentrar-se nos } \\
\text { cuidados pós-operatórios, quando a } \\
\text { maioria das LP se desenvolve. }\end{array}$ \\
\hline $\begin{array}{l}\text { O'Brien et al. } \\
\text { (Estados Unidos, } \\
2014)^{12}\end{array}$ & $\begin{array}{l}\text { Lesões por pressão } \\
\text { de estágios II, III e } \\
\text { IV, lesão tecidual } \\
\text { profunda e/ou } \\
\text { instável }\end{array}$ & $\begin{array}{l}\text { Foram eleitos } 2.695 \text { pacientes, dos quais } \\
288 \text { apresentaram lesões: } 261 \text { com LP tipo } \\
\text { II, } 22 \text { LP tipo III, } 10 \text { LP tipo IV, } 23 \text { LP tecidual } \\
\text { profunda e } 88 \text { LP tecidual instável. }\end{array}$ & $\begin{array}{l}\text { As LP no pós-operatório estavam } \\
\text { presentes em } 10,7 \% \text { dos pacientes graves. }\end{array}$ \\
\hline $\begin{array}{l}\text { Shaw et al. } \\
\text { (Taiwan, 2014) }^{13}\end{array}$ & $\begin{array}{l}\text { Lesão por pressão } \\
\text { estágio I }\end{array}$ & $\begin{array}{l}\text { Dos } 297 \text { pacientes que foram submetidos } \\
\text { a procedimentos cirúrgicos com mais } \\
\text { de } 30 \text { minutos sob raquianestesia ou } \\
\text { anestesia geral, } 29 \text { adquiriram LP estágio I } \\
\text { imediatamente após a cirurgia e } 15 \text { LP estágio } \\
\text { I após } 30 \text { minutos da cirurgia. }\end{array}$ & $\begin{array}{l}\text { Tipo da anestesia, pacientes com idade } \\
\text { avançada, tipo de cirurgia e posição } \\
\text { da cirurgia estão associados ao } \\
\text { desenvolvimento de LP. }\end{array}$ \\
\hline $\begin{array}{l}\text { Eteuati et al. } \\
\text { (Austrália, 2013) }{ }^{14}\end{array}$ & $\begin{array}{l}\text { Lesão de plexo } \\
\text { braquial }\end{array}$ & $\begin{array}{l}\text { Entre } 2005 \text { e } 2010 \text {, foram realizadas } 548 \\
\text { ressecções colorretais laparoscópicas, com } \\
\text { cinco casos de plexopatia braquial, por conta } \\
\text { do longo tempo de permanência na posição } \\
\text { de Trendelenburg. }\end{array}$ & $\begin{array}{l}\text { Para a prevenção da plexopatia } \\
\text { braquial, são recomendadas } \\
\text { as precauções: mudanças no } \\
\text { posicionamento do paciente, } \\
\text { principalmente com IMC baixo, } \\
\text { colocação na posição com ambos os } \\
\text { braços mantidos ao lado do tronco e } \\
\text { uso de coxins com gel ou almofadas } \\
\text { de espuma e travesseiros. }\end{array}$ \\
\hline $\begin{array}{l}\text { Jellish et al. (Estados } \\
\text { Unidos, 2013) }\end{array}$ & Lesão braquial & $\begin{array}{l}\text { Foi utilizado um monitoramento } \\
\text { somatossensitivo dos potenciais riscos } \\
\text { (PESS) para detectar lesões do plexo braquial, } \\
\text { relacionadas ao posicionamento durante } \\
\text { a cirurgia da base do crânio. Sessenta } \\
\text { e cinco pacientes, entre } 15 \text { e } 77 \text { anos, } \\
\text { foram estudados. A sensibilidade do PESS } \\
\text { para detecção de lesão foi de } 57 \% \text {, e a } \\
\text { especificidade, de } 94,7 \% \text {. }\end{array}$ & $\begin{array}{l}\text { Se houver ajuste na posição com } \\
\text { melhora nos PESS, a lesão do nervo } \\
\text { pode ser evitada. Assim, o monitor } \\
\text { pode ser usado para avaliação } \\
\text { rotineira do plexo braquial nos } \\
\text { procedimentos cirúrgicos ou, pelo } \\
\text { menos, nos pacientes obesos. }\end{array}$ \\
\hline $\begin{array}{l}\text { Mehta (Estados } \\
\text { Unidos, 2013) }\end{array}$ & $\begin{array}{l}\text { Queimadura química } \\
\text { e lesão térmica na } \\
\text { pele, nariz, boca, } \\
\text { lábios, traqueia, } \\
\text { faringe e tórax }\end{array}$ & $\begin{array}{l}\text { Coletadas } 5.194 \text { reclamações sobre } \\
\text { insucessos cirúrgicos, sendo } 103 \text { por } \\
\text { incêndios, e } 90 \% \text { dos incêndios foram } \\
\text { causados por eletrocautério. Das lesões } \\
\text { acometidas por eletrocautério, } 86 \% \text { ocorreram } \\
\text { na pele e } 10 \% \text { na boca. }\end{array}$ & $\begin{array}{l}\text { O reconhecimento da tríade de } \\
\text { fogo é crucial para evitar incêndios. } \\
\text { A educação continuada e a } \\
\text { comunicação entre os colaboradores } \\
\text { do CC e os protocolos de prevenção } \\
\text { de incêndio em procedimentos de alto } \\
\text { risco podem reduzir a ocorrência. }\end{array}$ \\
\hline
\end{tabular}

LP: Lesão por Pressão; IMC: índice de massa corporal; CC: centro cirúrgico. 
pressão, com maior evidência de aparição no pós-operatório, e queimaduras, que têm mais visibilidade no intraoperatório.

O tipo de cirurgia e o tempo operatório são fatores altamente relevantes para gerar traumatismo, contudo a equipe cirúrgica deve promover cuidados durante $\mathrm{o}$ ato operatório, desde o posicionamento adequado até o uso de acessórios para distribuir a pressão e proteger as proeminências ósseas. Quanto às queimaduras, a atenção é voltada ao uso do eletrocautério e aos componentes que podem levar ao início de incêndio na sala operatória.

Em relação ao objetivo inicial da pesquisa, verificaramse lacunas em relatos nos estudos para abordar as lesões que podem acontecer após o procedimento cirúrgico. Mesmo inserindo descritores que envolviam diferentes tipos de lesões, somente se localizaram artigos descrevendo lesões por pressão ou queimaduras, os quais atendiam aos critérios de inclusão.

Entender quais fatores geraram essas lacunas escapa do alcance dos pesquisadores, mas é notável que existem outras lesões que podem ser geradas por procedimentos cirúrgicos, sejam eles pelo posicionamento, sejam por itens químicos ou elétricos. Logo, há a necessidade de aprofundar a pesquisa, com a finalidade de intensificar as evidências diante do tema abordado.

\section{REFERÊNCIAS}

1. Lima AM, Sousa CS, Cunha ALSM. Patient safety and preparation of the operating room: reflection stud. J Nurs UFPE [Internet]. 2013 [acessado em 6 maio 2018];7(1):289-94. Disponível em: https://doi. org/10.5205/1981-8963-v7i1a10232p289-294-2013

2. Henriques AHB, Costa SS, Lacerda JS. Nursing care in surgical patient safety: an integrative review. Cogitare Enferm [Internet]. 2016 [acessado em 6 maio 2018];21(4):1-9. Disponível em: http:// dx.doi.org/10.5380/ce.v21i4.45622

3. Menezes S, Rodrigues R, Tranquada R, Müller S, Gama K, Manso T. Lesões decorrentes do posicionamento para cirurgia: incidência e fatores de risco. Acta Med Port [Internet]. 2013 [acessado em 6 maio 2018];26(1):12-6. Disponível em: https://www.actamedicaportuguesa. com/revista/index.php/amp/article/viewFile/4006/3204

4. Associação Brasileira de Enfermeiros de Centro Cirúrgico, Recuperação Anestésica e Centro de Material e Esterilização. Diretrizes de práticas em enfermagem cirúrgica e processamento de produtos para a saúde. 7ª ed. São Paulo: SOBECC; Barueri: Manole; 2017.

5. Barbosa MH, Oliva AMB, Sousa Neto AL. Ocorrência de lesões perioperatórias por posicionamento cirúrgico. Rev Cubana Enferm [Internet]. 2011 [acessado em 6 maio 2018];27(1):31-41. Disponível em: http://www.medigraphic.com/pdfs/revcubenf/cnf2011/cnf111e.pdf

6. Lopes CMM, Haas VJ, Dantas RAS, Oliveira CG, Galvão CM. Escala de avaliação de risco para lesões decorrentes do posicionamento cirúrgico. Rev Latino-Am Enferm. 2016;24:e2704. http://doi. org/10.1590/1518-8345.0644.2704

7. Domingos CMH, lida LIS, Poveda VB. Glycemic control strategies and the occurrence of surgical site infection: a systematic review. Rev Esc Enferm USP. 2016;50(5):868-74. http://doi.org/10.1590/ s0080-623420160000600022

8. Santos CMC, Pimenta CAM, Nobre MRC. A estratégia PICO para a construção da pergunta de pesquisa e busca de evidências. Rev Latino-Am Enferm. 2007;15(3):508-11. http://dx.doi.org/10.1590/ S0104-11692007000300023
9. Steyer NH, Oliveira MC, Gouvêa MRF, Echer IC, Lucena AF. Clinical profile, nursing diagnoses and nursing care for postoperative bariatric surgery patients. Rev Gaúcha Enferm. 2016;37(1):e50170. http:// dx.doi.org/10.1590/1983-1447.2016.01.50170

10. Jalali SM, Moradi M, Khalaj A, Pazouki A, Tamannaie Z, Ghanbari S. Assessment of electrosurgery burns in cardiac surgery. Trauma Mon. 2015;20(4):e18996. https://dx.doi.org/10.5812\%2Ftraumamon.18996

11. Hayes RM, Spear ME, LeeSI, Krauser Lupear BE, Benoit RA, Valerio R, et al. Relationship between time in the operating room and incident pressureulcers: a matched case-control study. Am J Med Qual [Internet]. 2015 [acessado em 5 maio 2018];30(6):591-7. http://doi.org/10.1177/1062860614545125

12. O'Brien DD, Shanks AM, Talsma A, Brenner PS, Ramachandran SK. Intraoperative risk factors associated with postoperative pressure ulcers in critically Ill patients: a retrospective observational study. Crit Care Med [Internet]. 2014 [acessado em 5 maio 2018];42(1):40-7. http://doi. org/10.1097/CCM.0b013e318298a849

13. Shaw LF, Chang PC, Lee JF, Kung HY, Tung TH. Incidence and predicted risk factors of pressure ulcers in surgical patients: experience at a Medical Center in Taipei, Taiwan. Biomed Res Int [Internet]. 2014 [acessado em 5 maio 2018];2014:416896. http://doi.org/10.1155/2014/416896

14. Eteuati J, Hiscock R, Hastie I, Hayes I, Jones I. Brachial plexopathy in laparoscopic-assisted rectal surgery: a case series. Tech Coloproctol. 2013;17(3):293-7. http://doi.org/10.1007/s10151-012-0920-8

15. Jellish WS, Sherazee G, Patel J, Cunanan R, Steele J, Garibashvili K, et al. Somatosensory evoked potentials help prevent positioning-related brachial plexus injury during skull base surgery. OtolaryngolHead Neck Surg. 2013;149(1):168-73. http://doi.org/10.1177/0194599813482878

16. Mehta SP, Bhananker SM, Posner KL, Domino KB. Operating room fires: a closed claims nalysis. Anesthesiology. 2013;118(5):1133-9. http://doi.org/10.1097/ALN.0b013e31828afa7b

17. Hart SR, Yajnik A, Ashford J, Springer R, Harvey S. Operating room fire safety. Ochsner J [Internet]. 2011 [acessado em 5 maio 2018];11(1):37-42. Disponível em: https://www.ncbi.nlm.nih.gov/pmc/articles/PMC3096161 